\title{
2-D Terrain Chart Mapping for a Navigation Robot using Tactile Sensor for System Modelling
}

\author{
M. Utsav and R. Gomathi Bhavani \\ Department of Electrical and Electronics Engineering, \\ Birla Institute of Technology and Science Pilani, Dubai Campus, Dubai, UAE
}

\begin{abstract}
Robotics is a vast, inter-disciplinary field in which various principles and methods of control system are employed for basic operation and effectiveness of control. This study presents the modelling of a navigation robot set in an adaptive feedback control system. The simulation of control system model involved the two-dimensional terrain mapping of a certain region with the aid of a tactile sensor. The tactile sensor designed as a model for the calculation was based on the mechanism of a TekScan ForceFlex Sensor A201 which has a linear pressure output for the force applied and uses Mylar as a substrate. The basic model aims to utilize the pressure difference to calculate the height difference between the ground level and the test material, thus, enabling the charting of a suitable terrain map of a geographical region. In this model, fuzzy logic has been used to create a tentative map of the relationship between pressure-voltage difference and height and adaptive neuro fuzzy inference system in particular has been used to validate the model. With a testing error of 0.0028852 , results indicate that the model was able to capture the input-output mapping well.
\end{abstract}

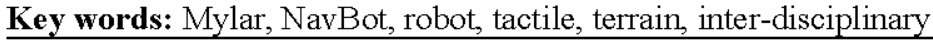

\section{INTRODUCTION}

Robotics is a vast, inter-disciplinary field with applications in various industries such as medical, education and military training. Though several control system methods such as predictive, model-based or model-free control are applied in this field for basic operation and effectiveness of control, the two kinds are frequently employed; robust and adaptive as they both employ feedback systems for data collection and control. Though several advanced systems have been reported in this field such as biomimetic sensors (Wettels et al., 2009; Landau et al., 2011), a lot of potential is still left untapped.

The major impediment with these sophisticated systems is their high costs of fabrication and complexity of design. The main objective of this study is to demonstrate the modeling of a simple, low-cost system for terrain mapping with an adaptive feedback control system. A review of the literature reveals the existence of a limited number of studies on 2-D terrain chart mapping for a Navigation Robot (NavBot).

Literature review: Some of the prominent works done in the areas of mapping and obstacle detection of robots are discussed in the literature. Wolf et al. (2005) presented a new approach for terrain mapping and classification using mobile robots with $2 \mathrm{D}$ laser range finders. The maps generated by this approach can be used for local obstacle avoidance, path planning and navigation, detection of changes in the terrain and object recognition (Wolf et al., 2005). Schadler et al. (2014) proposed methods for efficient 3D mapping and real-time $6 \mathrm{D}$ pose tracking of autonomous robots using a continuously rotating $2 \mathrm{D}$ laser scanner. Terrain drivability was assessed within a global environment map and used for planning feasible paths and the approach was evaluated using challenging real environments (Schadler et al., 2014). Nam et al. (2017) aimed to present a localization method based in cooperation between aerial and ground robots in an indoor environment. The proposed method allowed a ground robot to reach accurate destination by using a 2.5D elevation map which was formed by projecting height information of an obstacle using depth information obtained by the red green and blue-depth sensor onto a grid map which was generated by using the 2D Laser sensor and scan matching (Nam et al., 2017). Kweon (1991) developed 3-D vision techniques for incrementally building an accurate 3-D representation of rugged terrain using 3-D information acquired from multiple sensors. This research developed the Locus method to model the rugged terrain. Tian (2016) proposed a strategy based on

Corresponding Author: R. Gomathi Bhavani, Department of Electrical and Electronics Engineering, Birla Institute of Technology and Science Pilani, Dubai Campus, Dubai, UAE 
the relative position of a robot to a boundary compared to other robots with good performances in total exploration time compared to the existing strategies. The objective of the research (Wells, 2006) was to perform and analyse a method of terrain mapping with a tracked robot. Ye and Borenstein (2003) introduced a new terrain mapping method for mobile robots with a 2-D laser rangefinder. Gomez (2015) proposed a map building method based on sets that can solve large-scale problems and a method to navigate more efficiently in unknown environments. The following section explains the materials and methodologies that have been taken into consideration for modeling the system.

\section{MATERIALS AND METHODS}

In order to achieve and maintain a requisite level of performance of the control system when the parameters of the plant are unknown and/or change with time or environmental parameters, the set of techniques that go into automated adjustment of the controllers in real time is called adaptive feedback control. In this model, the change in the model navigation as the terrain of the land changes requires an adaptive feedback network.

There exists a linear relation between pressure and height. This can be proved via. the relation observed between stress and strain. Strain can be described as a deformation (permanent or temporary) of solid substances caused by stress (pressure). For elastic systems, there exists a direct correlation between the two quantities, till the elastic point is reached, at which point, there is nonlinearity as can be seen in Fig. 1.

The use of an elastic substrate in a sensor as a model material ensures that the elastic point is not achieved as that would result in damage of the sensor as the accuracy of pressure reading guarnatees readings with negligible errors.

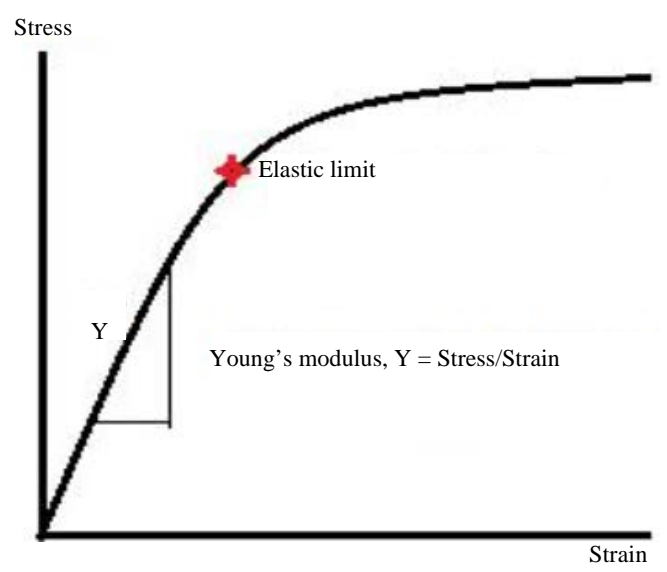

Fig 1: Stress strain curve for elastics
The system described in this study was simulated in two steps. The first step involved creation of a fuzzy network with three membership functions (namely ground, medium and high) for each height level, so as to estimate a tentative idea of the output (height) dependence on input functions (pressure or voltage difference) using the OR relation between the two inputs. For the sake of convenience, only the pressure input has been used in this scenario for the output readings as the voltage difference is directly dependent on pressure difference in a sensor. The second step involved the validation of the model and relationship between the parameters using Adaptive Neuro Fuzzy Inference System (ANFIS). Some researches by Jang (1993), Jang and Mizutani (1996), Jang et al. (1997) and Petkovic et al. (2012) have demonstrated the employability and suitability of ANFIS in systems that have a non linear, uncertain or complex relationship between inputs and output.

Modelling of primary fuzzy network: The primary network was simulated using either the voltage difference or the pressure difference as an input. Pressure sensors often provide the output in terms of voltage readings, thus, the unit volt is taken into consideration while creating the architecture. The surface map shown in Fig. 2 indicates the direct relation between either of the inputs with the output for the three surface levels mentioned above. As can be noticed, low input values signify ground levels while medium values correspond to medium level and high values for the higher levels. The flat surface areas between the levels have just been taken for ease of differentiation. Out of the nine rules (three pressure levels $\times$ three voltage levels) that were created with the $\mathrm{OR}$ function, only three were considered as they more closely represented the linear dependence of height for each of the three levels.

Modelling of ANFIS architecture: ANFIS stands for adaptive neuro fuzzy inference system and belongs to

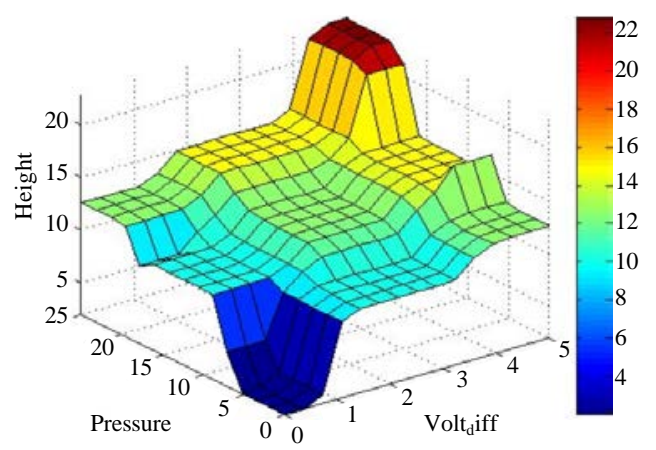

Fig. 2: Surface for voltage difference-pressure and height 
the class of hybrid intelligent approach, used for modeling and validating system dynamics in which fuzzy logic and neural networks complement each other. The use of hybrid intelligent techniques brings to the table the advantages of tackling uncertainty, vagueness and also catering to high-dimensionality. Typically, the neural network is mixed with fuzzy inference systems in three ways, namely cooperative, concurrent and fused. The most common architecture is the fused Neuro Fuzzy System (NFS) that uses ideas of neural networks just to learn some internal parameters of a fixed structure (Nauck et al., 1997). The ANFIS belongs to fused NFS and it was introduced by Jang (1992) and is able to approach any linear or non linear function (universal approximator) (Jang, 1993). ANFIS can represent structured knowledge and the model structure need not be known prior (Jang, 1993). The synergism in ANFIS allows to incorporate human knowledge effectively, deal with imprecision and uncertainty and learn to adapt to unknown or changing environment for better performance (Jang et al., 1997). The attractive features of ANFIS include: easy to implement, fast and accurate learning, strong generalization abilities, excellent explanation facilities through fuzzy rules and easy to incorporate both linguistic and numeric knowledge for problem solving (Jang et al., 1997).

ANFIS architecture is directly applicable to robotic control due to adaptive capability, learning ability, structured knowledge representation, better integration with other control design approaches and flexibility of parallel operation (Bhavani and Khan, 2010). The adaptive capability of ANFIS was also demonstrated in the corresponding researchers earlier work involving non linear control scheme (Bhavani and Khan, 2009).

In this research, the inverse-ANFIS function was used to create the values based on the output data obtained. The ANFIS relation thus obtained for the system is shown in Fig. 3. Due to the lack of human expertise in the given problem, initial membership

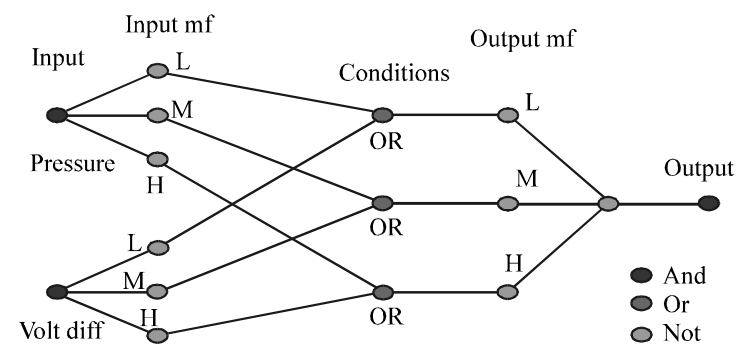

Fig. 3: Fuzzy inference system generated by ANFIS functions were set up by intuition and the learning process was initiated, so that, a set of fuzzy, if then, rules that approximate the data set could be generated. Computations in ANFIS effectively tune the membership functions, so that, output error is minimised. The membership functions take their final forms after training. This automatic generation of data-driven rules and parameter adjustment makes ANFIS a good candidate for adaptive control systems. At the same time, ANFIS requires smaller number of parameters and hence, converges faster to a control decision which makes a good case for real time implementation.

The relation between pressure and height was developed using the following steps: for the sake of reducing the complexity of obtaining the variables, only pressure was considered out of the two probable outputs. In the following discussion, the following variables are assumed: $\mathrm{L}=$ Highest reading for $\mathrm{a}$ given area, 1 = Percentage value for a given area $(\% \mathrm{~L})$, $\Delta \mathrm{LO}=$ Difference of original value $\Delta \mathrm{L}^{\prime}=$ New length difference, then the pre-existing equation for Young's modulus $\mathrm{Y}$ can be written as:

$$
\begin{gathered}
\mathrm{Y}=\frac{\text { Stress (Pr essure) }}{\text { Strain }(\text { Deformation })} \\
P=Y\left(\frac{\Delta L}{L}\right) \\
P-P 0=Y\left(\frac{\Delta L^{*}}{L}\right) \\
P=P 0+Y\left(\frac{\Delta L^{*}}{L}\right) \\
P=\frac{Y}{L}(\Delta L 0-\Delta L) \\
P=\frac{Y}{L}\left(1_{0}-L+1-L\right)
\end{gathered}
$$

Taking the original value $1_{0}$ as zero, since, it's the initial reading:

$$
\begin{aligned}
& P=\frac{Y}{L}(1-2 L) \\
& P=Y\left(\frac{1}{L}-2\right)
\end{aligned}
$$


Table 1: Sample checking and training values for ANFIS Pressure (GPa)

\begin{tabular}{lrr} 
& \multicolumn{1}{c}{$\mathrm{Si}$} & Mylar \\
\hline 0.0 & 0.00 & 0.00 \\
0.1 & 15.00 & 0.52 \\
0.2 & 30.00 & 1.05 \\
0.5 & 75.00 & 2.62 \\
0.7 & 105.00 & 3.67 \\
1.0 & 150.00 & 5.24 \\
1.1 & 165.00 & 5.76 \\
1.2 & 180.00 & 6.29 \\
1.5 & 225.00 & 7.86 \\
2.0 & 300.00 & 10.48 \\
\hline
\end{tabular}

However, the readings were found to be negative due to zero initial error. The lowest value $(1=0), \mathrm{P}=-2 \mathrm{Y}$ and highest $(1=1), \mathrm{P}=-\mathrm{Y}$. To overcome this error, $2 \mathrm{Y}$ was added to Eq. 9:

$$
\mathrm{P}=\mathrm{Y}\left(\frac{1}{\mathrm{~L}}-2+2\right)=\mathrm{Y} \frac{1}{\mathrm{~L}}
$$

Using the .dat format files created from excel databases, a set of readings were created which were then entered into the ANFIS GUI as training data and checking data to create values for input-output mapping. A sample set of values of training and checking data are shown in Table 1 for $\mathrm{L}=7 \mathrm{~cm}$.

Values for both Mylar ( $\mathrm{Y}=5.24 \mathrm{GPa}$ ) and Silicon ( $\mathrm{Y}=150 \mathrm{GPa}$ ) were taken into consideration as they are currently the popular choices for pressure sensor substrates (Petkovic et al., 2012). Mylar was the substrate for the pressure sensor model TekScan ForceFlex A201 used in the work that proposed a novel system and algorithm called gait classifier of pressure centers to sense the pressure distribution in partial weight bearing (Lin et al., 2013).

The height $\mathrm{L}$ is the highest reading that can be possibly obtained for a given area. It can also be taken as a reference value which could be assumed as unity in some cases, so as to obtain values in terms of percentages for a given surface in scenarios where the height of the highest point remains unknown. These values when fed in simulation software such as MATLAB, provide an idea of the topographical landscape of a system.

\section{RESULTS AND DISCUSSION}

Figure 4 represents the system schematic to assess the initial idea of terrain mapping for a given area. The pressure readings, so, obtained for each unit distance recorded by the on-board odometer from the sensor model
Table 2: Sample data values from odometer reading

\begin{tabular}{|c|c|c|}
\hline Distance (m) & Pressure (GPa) & Height (cn \\
\hline 1 & 0.05 & 0.07 \\
\hline 2 & 4.77 & 6.37 \\
\hline 3 & 4.45 & 5.95 \\
\hline 7 & 1.57 & 2.10 \\
\hline 8 & 1.78 & 2.38 \\
\hline 9 & 3.98 & 5.32 \\
\hline 10 & 2.31 & 3.08 \\
\hline 15 & 3.41 & 4.55 \\
\hline 17 & 3.30 & 4.41 \\
\hline 19 & 3.30 & 4.41 \\
\hline 20 & 1.15 & 1.54 \\
\hline $\begin{array}{l}\text { Odometer: distance traversed } \\
\text { detected by on-board odometer }\end{array}$ & \multicolumn{2}{|c|}{$\begin{array}{l}\text { Pressure sensor: } \\
\text { pressure difference } \\
\text { detection in } \mathrm{Pa} \text { or } \mathrm{V}\end{array}$} \\
\hline \multicolumn{3}{|l|}{$\downarrow$} \\
\hline $\begin{array}{c}\text { The corresponding height } \\
\text { difference observed from } \\
\text { Table } 1\end{array}$ & \multicolumn{2}{|c|}{$\begin{array}{l}\text { The height values for each } \\
\text { distance recorded sent for } \\
\text { terrain mapping }\end{array}$} \\
\hline \multicolumn{3}{|c|}{$\sqrt{ }$} \\
\hline $\begin{array}{c}\text { The idea of terrain obtained } \\
\text { for a given area }\end{array}$ & & \\
\hline
\end{tabular}

Fig. 4: System schematic

as shown in step 1 can be equated to the subsequent height value from Table 1. This table value can be recorded for the corresponding distance reading.

After the model was input with training and checking data sets, the result was obtained. The relation between pressure and height was observed as shown in Fig. 5 wherein blue dotted lines represent training data while red indicate checking data points. The graph showed a testing error of 0.0028852 which indicates that the model was able to capture the input-output mapping quite well. A model with 40 epochs was obtained with training error of 0.0028619 as observed in Fig. 6. This validates the linear relation between the pressure and height that was assumed earlier.

Table 2 presents a sample set of data, created randomly from odometer reading. The values provided are very abstract and are just sample readings. The length of the highest point was taken as $\mathrm{L}=7 \mathrm{~cm}$ and the height found was corresponding to the values provided in Table 1 pressure values. Figure 7 shows the sample terrain graph for a given area (measuring in total, $1.4 \mathrm{~m}^{2}$ ). The non linear terrain mapping can be observed which necessitated model capture and analysis. The $\mathrm{X}$-axis is the distance in metres and $\mathrm{Y}$-axis is the height in $\mathrm{cm}$. The graph obtained matches closely with the values assumed, thus, validating the correctness of model built. 


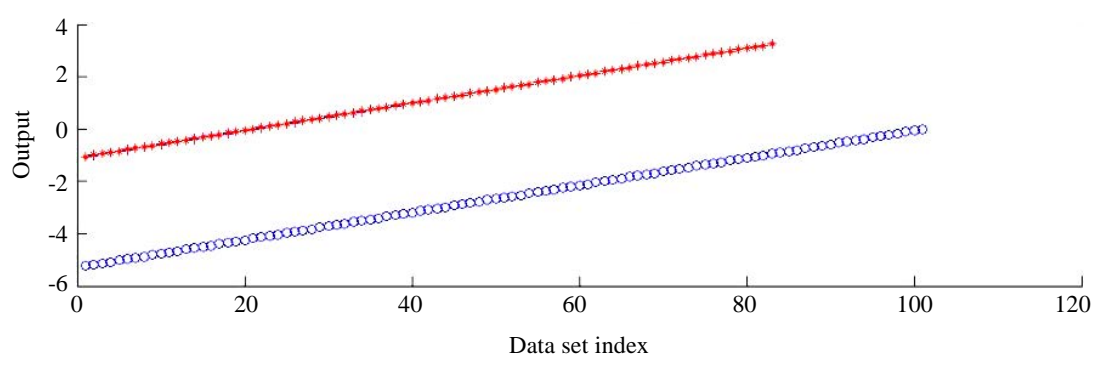

Fig. 5: Training and checking graph for Mylar (Rred: checking data and Blue: training data, 000)

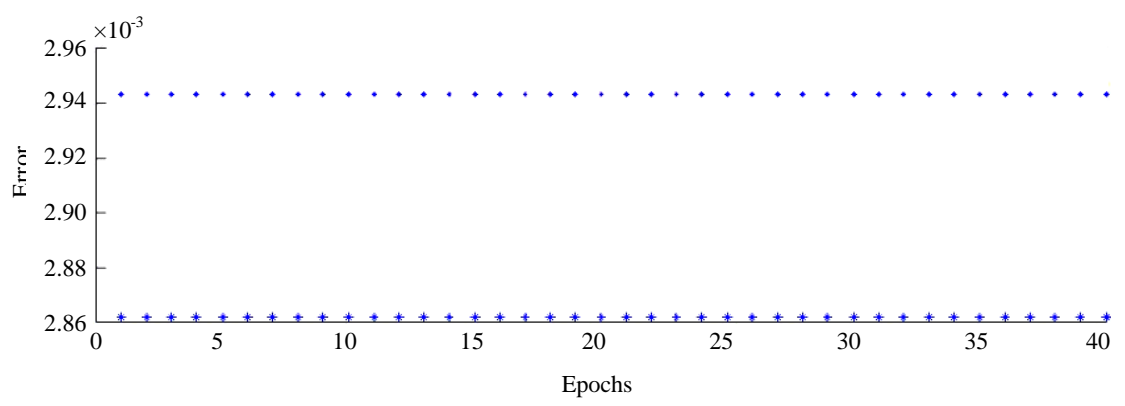

Fig. 6: Error checking graph trained for 40 epochs (training error)

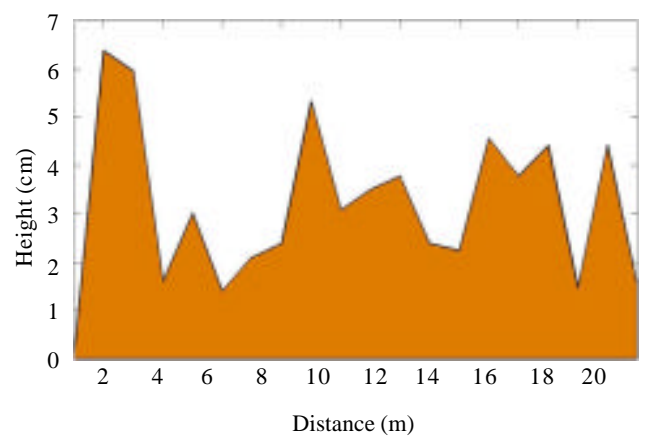

Fig. 7: Terrain graph

\section{CONCLUSION}

In this research, a model for terrain mapping for an automated robot was presented which utilized the linear relation between stress and strain. Primary fuzzy relations and ANFIS were used to validate the relationship between input and output graphs. The biggest advantage of the NavBot is its cost-efficiency and ease of design and holds the potential for many applications (Sanderson et al., 1987). The future scope of such systems could be in pipelines that are too thin to accommodate devices with bigger structures and higher number of detectors (sonar, lidar), wherein these mechanisms can be used as a part of pipeline damage repair systems, since, they require minimal space for operation. With the increase in infrastructure and accessibility for, specially, abled citizens all over the world, implementation of this system architecture under canes for the blind can enable the data recognition readings to be transmitted via. bluetooth headsets, thus, making them navigate pathways easily as well as find misplaced objects.

\section{ACKNOWLEDGEMENT}

The researchers would like to acknowledge and thank the management of Birla Institute of Technology and Science Pilani, Dubai Campus, for facilitating this research and its software implementation.

\section{REFERENCES}

Bhavani, R.G. and M.A. Khan, 2009. An intelligent simulation model for blind position control in daylighting schemes in buildings. Building Simulation, 2: 253-262.

Bhavani, R.G. and M.A. Khan, 2010. Intelligent controllers for integrated lighting schemes: Model based approach combining simulator and learning. Intl. J. Simul. Syst. Sci. Technol., 11: 33-40.

Gomez, E.Z., 2015. Map-building and planning for autonomous navigation of a mobile robot. Ph.D Thesis, Department of Automatic Control, Center for Research and Advanced Studies of the National Polytechnic Institute, Mexico. 
Jang, J.S., 1993. ANFIS: Adaptive-network-based fuzzy inference system. IEEE. Trans. Syst. Man Cybern., 23: 665-685.

Jang, J.S.R. and E. Mizutani, 1996. Levenberg-Marquardt method for ANFIS learning. Proceedings of the Conference on North American Fuzzy Information Processing Society, June 19-22, 1996, IEEE, New York, USA., ISBN:0-7803-3225-3, pp: 87-91.

Jang, J.S.R., 1992. Neuro-fuzzy modeling: Architecture, analyses and applications. Ph.D Thesis, Department of Electrical Engineering and Computer Science, University of California, Berkeley, USA.

Jang, J.S.R., C.T. Sun and E. Mizutani, 1997. Neuro-fuzzy and Soft Computing: A Computational Approach to Learning and Machine Intelligence. Prenctice Hall International, New Jersey, USA., ISBN:9780132874670, Pages: 614.

Kweon, I.S., 1991. Modeling rugged terrain by mobile robots with multiple sensors. Ph.D Thesis, Carnegie Mellon University, Pittsburgh, USA.

Landau, I.D., R. Lozano, M. M'Saad and A. Karimi, 2011. Adaptive Control: Algorithms, Analysis and Applications. 2nd Edn., Springer, London, UK., ISBN:9780857296641, Pages: 590.

Lin, S.W., C.C. Tuan, T.F. Lee, C.H. Lu and C.H. Chang et al., 2013. Recognition of gait patterns with partial weight bearing using insole plantar pressure sensor. Proceedings of the 3rd IASTED Asian Conference on Modelling, Identification and Control, AsiaMIC Vol. 2013, April 10-12, 2013, Phuket, Thailand, pp: 14-20.

Nam, T., J. Shim and Y. Cho, 2017. A 2.5 D map-based mobile robot localization via cooperation of aerial and ground robots. Sens., 17: 2730-2753.

Nauck, D., F. Klawonn and R. Kruse, 1997. Foundations of Neuro-Fuzzy Systems. John Wiley and Sons, New York, ISBN-13: 9780471971511, Pages: 316.
Petkovic, D., M. Issa, N.D. Pavlovic, N.T. Pavlovic and L. Zentner, 2012. Adaptive neuro-fuzzy estimation of conductive silicone rubber mechanical properties. Expert Syst. Appl., 39: 9477-9482.

Sanderson A.C., L.E. Weiss and C.P. Neuman, 1987. Dynamic sensor-based control of robots with visual feedback. IEEE Trans. Robot. Automat. RA, 3: 404-417.

Schadler, M., J. Stuckler and S. Behnke, 2014. Rough terrain 3D mapping and navigation using a continuously rotating $2 \mathrm{D}$ laser scanner. KI Kunstliche Intelligenz, 28: 93-99.

Tian, D., 2016. Cooperative mobile robots optimal mapping and navigation. Ph.D Thesis, Ecole Centrale de Lille, France.

Wells, D., 2006. Terrain mapping with a tracked robot. MSc Thesis, The University of Western Australia, Crawley, Austarlia.

Wettels, N., A.R. Parnandi, J.H. Moon, G.E. Loeb and G.S. Sukhatme, 2009. Grip control using biomimetic tactile sensing systems. IEEE. ASME. Trans. Mechatron., 14: 718-723.

Wolf, D.F., G.S. Sukhatme, D. Fox and W. Burgard, 2005. Autonomous terrain mapping and classification using hidden markov models. Proceedings of the 2005 IEEE International Conference on Robotics and Automation, April 18-22, 2005, IEEE, Barcelona, Spain, pp: 2026-2031.

Ye, C. and J. Borenstein, 2003. A new terrain mapping method for mobile robots obstacle negotiation. Proceedings of the International Conference on Unmanned Ground Vehicle Technology Vol. 5083, April 21-25, 2003, International Society for Optics and Photonics, AeroSense, Orlando, Florida, USA., pp. 52-63. 